\title{
La gestión institucional y su efecto en el logro de la calidad académica. Caso: Escuela Académico Profesional de Ingeniería Electrónica de la Facultad de Ingeniería Electrónica y Eléctrica de la Universidad Nacional Mayor de San Marcos. Año 2020
}

Institutional management and its effects on academic quality

achievement. Case: Electronic Engineering School of Electronic and

Electric Engineering Faculty of Universidad Nacional Mayor de San

Marcos. Year 2020

\author{
Nolan Jara Jara ${ }^{1}$, Gladys María Garay Capcha ${ }^{2}$ \\ ${ }^{1}$ Universidad Nacional Mayor de San Marcos \\ ${ }^{2}$ Universidad César Vallejo
}

\begin{abstract}
Resumen
Una de las estrategias educativas que cada institución debería poseer es saber identificar los estilos de gestión de cada unidad, ya que estos pueden influir directamente en lograr un buen rendimiento académico. El objetivo de la investigación fue Determinar el efecto de la aplicación de la gestión institucional en el logro de indicadores de calidad del área académica universitaria de la Escuela Académica Profesional de Ingeniería Electrónica de la Facultad de Ingeniería electrónica y eléctrica de la Universidad Nacional Mayor de San Marcos, en el año 2020. Al aplicar un diseño no experimental descriptivo correlacional, se seleccionó una muestra no probabilística intencionada de 70 docentes. $\mathrm{Al}$ respecto se aplicó dos encuesta-cuestionario, una acerca de la Gestión Institucional y otra sobre Calidad del área académica universitaria. Los resultados fueron procesados aplicando el programa estadístico SPSS versión 20.0. Como consecuencia de la exploración estadística de los resultados obtenidos se concluye que es significativo el efecto del uso de la gestión universitaria en la consecución de la calidad académica universitaria en la Escuela Académica Profesional de Ingeniería Electrónica de la Facultad de Ingeniería electrónica y eléctrica de la Universidad Nacional Mayor de San Marcos, en el año 2020.
\end{abstract}


Palabras clave: gestion institucional; calidad de la educación; educación superior; área académica universitaria.

\begin{abstract}
One of the educational strategies that each institution should have is to know how to identify each unit's management styles, since these could directly influence in achieving a high academic performance. The aim of this research was to determine the effect of the application of institutional management in achieving academic quality indicators in the Electronic Engineering school from the Electronic Engineering Faculty of Universidad Nacional Mayor de San Marcos in 2020 by applying a correlational descriptive non experimental design, an intended non probabilistic sample consisting of 70 professors was selected. 02 questionnaire-surveys were applied to the effect, one on institutional management and the other on university academic quality. The results were processed by the statistical software SPSS v. 20.0. As a consequence of the statistical exploration of the results, it is concluded that the effect of university management on achieving university academic quality in the Electronic Engineering school from Universidad Nacional Mayor de San Marcos in 2020 is significant.
\end{abstract}

Keywords: institutional management; quality of education; higher education; university academic area.

\section{Introducción}

Frente a los rápidos cambios y los evidentes resultados del fenómeno de la globalización, las universidades han observado que es necesario acondicionar sus procedimientos para acomodarse a los modernos acontecimientos del escenario educativo, y así poder satisfacer su misión. Ello debe motivar su conversión en instituciones que se perfeccionan y se instruyen a partir de su correspondiente pericia y actuación, para así poder conseguir su continuidad en la colectividad por medio de una gestión institucional sustentada en la excelencia de la calidad. El actual estudio ha tenido como objetivo determi- nar el efecto de la aplicación de la gestión institucional en el logro de la calidad del área académica universitaria en la Escuela Académica Profesional de Ingeniería Electrónica de la Facultad de Ingeniería electrónica y eléctrica de la Universidad Nacional Mayor de San Marcos, en el año 2020.

Según Olguín y Fallas (2020), el proceso encaminado a enriquecer los proyectos educativos en las instituciones promueve la autonomía institucional en el marco de las políticas públicas y nos muestra una línea de estudio trascendental que beneficia el afianzamiento de las organizaciones educativas tanto en educación básica como en educación superior, en el sector 
universitario. Asimismo, se tiene una ocasión de mejoramiento del desarrollo de los procedimientos de gestión administrativa, académica y de rastreo de los estudiantes inscritos en cada una de las carreras profesionales.

Según Rodríguez y Tardo (2018), el más importante efecto de la gestión institucional para mejorar la calidad de la formación profesional universitaria, en el período 2015-2017, empleando indicadores pertinentes, son los siguientes:

El progreso científico- metodológico del ejercicio docente en las instituciones educativas universitarios y el desarrollo de las actividades educativas en el pregrado, a partir de la preparación en los temas de las contribuciones teórico - prácticos conseguidos a consecuencia de las capacitaciones académicos (diplomados, post grados, segunda especialización): modelos, estrategias, metodologías, procedimientos, modificaciones a los programas, asignaturas optativas, entre otros.

Según Razo, Iñigo y Dibut (2018) los indicadores de valoración del efecto de la gestión institucional para la excelencia en la formación de los nuevos profesionales, el progreso en la gestión institucional de la educación superior y su codificación deben sostenerse en los principios fundamentales de procedimientos universalmente comprobados, identificados y aprovechado en el entorno determinado de la institución educativa que se trate siempre de dirigir al logro de la mejora continua de su calidad y pertinencia.

Según Rubio y Zamora (2018), en referencia a la UNESCO (2015), se ha permitido establecer que la calidad de la Educación Superior es un tema valioso, y que se despliega en el entorno de una ideología de la búsqueda y comprobación del perfeccionamiento continuo de la gestión de los procedimientos universitarios y sus consecuencias.

Según Sovero (2007) se considera que la gestión institucional "alude al cúmulo de procedimientos y labores de dirección de las diferentes actividades administrativas que proporcionan un soporte a la gestión pedagógica". De igual forma, indica como actividades primordiales de dirección a la planificación, organización, comunicación, control y participación. Igualmente, manifiesta que la gestión institucional debe fundamentarse en las siguientes habilidades:

Liderazgo: pensado como la habilidad para establecer que los colaboradores que conforman parte de la institución actúen, con la finalidad de conseguir el cumplimiento de los propósitos institucionales.

Reingeniería: concebida como una habilidad para renovar, redireccionar o reestructurar las actividades o procedimientos educativos con la finalidad de optimizar la calidad de la educación. A la reingeniería también se le puede considerar como innovación.

Dirección con creatividad: asunto que conlleva que el director y el comité directivo deben ser ingeniosos en la ejecución de la gestión institucional.

Según Guevara (2003) la gestión consiste en liderar, monitorear, decidir, promover y administrar el conjunto de procesos en la conducción y manejo de todos los recursos para lograr fines y objetivos establecidos dentro del proyecto de desarrollo de la Institución que gestiona. A continuación, algunos modelos de gestión: 
a) gestión estratégica: es la que analiza las fortalezas, potencialidades, debilidades, limitaciones.

b) gestión operativa: es la que prevé las acciones presentes y objetivos futuros.

c) gestión del proceso educativo: está enmarcado en actividades de elaboración de programas educativos del proceso enseñanza- aprendizaje.

d) gestión de las actividades administrativas como: planificación, presupuesto, abastecimiento, racionalización, personal, estadística, contabilidad, jurídico legal, información y comunicaciones, tesorería, control y evaluación.

e) gestión de recursos: se diversifica teniendo en cuenta humanos, docentes, administrativos, salud y apoyo, materiales, bienes educativos, financieros, recursos monetarios y tecnológicos, funciones técnico- administrativas, tiempo; implica organización y eficiencia.

Martínez (2000), sobre la gestión institucional universitaria, menciona que la gestión institucional se sustenta en el concepto de institución, que excede la idea de corporación para predominar la exploración de las normativas, reglamentos, procedimientos esperados, régimen de incentivos, dispositivos de vinculación con la sociedad, entendimientos, autenticidades, etc. La institución universitaria mantiene una personalidad especialmente opinante para la exploración y la apreciación de la gestión. La universidad es un mundo semiológico, con narraciones que coadyuvan a conceder significado a la existencia, pero que al mismo tiempo la edifican y la concretizan, dirigiendo el razonamiento y sistematizando el modo de vida diaria.

Sovero (2007), en referencia a De la Orden (1991), expresa que la calidad de la educación es la consecuencia de la relación que se obtiene entre los aspectos básicos, internos y externos de la estructura de la institución educativa; procedimientos, métodos didácticos y cultura organizacional.

Facundo (2009) menciona que la calidad está relacionada con el requerimiento de la sociedad y en ese escenario una formación académica de calidad con la finalidad de hacer posible el cumplimiento de las obligaciones de educación que se propone en la sociedad a la que se atiende. Por lo tanto, una educación puede ser de calidad en determinado tiempo y lugar, pero no necesariamente en cualquier momento y lugar.

\section{Materiales y Métodos}

En esta investigación de carácter científico se contemplan dos variables: Gestión institucional universitaria y Calidad del área académica universitaria.

Método: El estudio de la presente investigación es ex post facto. Es decir, es una búsqueda sistemática empírica, en la cual el científico no tiene control directo sobre las variables independientes, porque ya acontecieron sus manifestaciones o por ser intrínsecamente no manipulables. Se hacen inferencias sobre las relaciones de 
ellas, sin intervención directa, a partir de la variación concomitante de las variables independientes y dependientes.

Enfoque: Por el enfoque del estudio de las variables, esta es cuantitativa.

El enfoque utilitario predominante es teorético o especulativo.

Tipo: El presente trabajo es de tipo descriptivo, se reseñan las características o rasgos de la situación o fenómeno del objeto de estudio.

Por el tipo de interrogante suscrita en este estudio, es teórico descriptivo relacional no causal.

Diseño: Se considera un diseño no experimental, descriptivo correlacional; por cuanto este tipo de estudio "implica la recolección de dos o más conjuntos de datos de un grupo de sujetos con la intención de determinar la subsecuente relación entre estos conjuntos de datos".

\section{Población y muestra}

Población: En la presente investigación la población escogida para el análisis estadístico del estudio ha estado integrada por los docentes de la Carrera Profesional de Ingeniería Electrónica, de la Facultad de Ingeniería electrónica y eléctrica de la UNMSM en el año 2020, conforme se detalla en el siguiente cuadro.

Muestra: Teniendo en cuenta que la población de profesores de la FIEEUNMSM no es grande, la muestra de los docentes ha sido no probabilística intencionada, dicha representatividad se da en base a una opinión o intención particular de quien selecciona la muestra.

\begin{tabular}{ll}
\hline Escuelas AP de IE-UNMSM & Docentes \\
\hline Doc. Principales & 10 \\
Doc. Asociados & 33 \\
Doc. Auxiliares & 14 \\
Doc. Pefes Práctica & 04 \\
Doc. Contratados & 09 \\
\hline TOTAL & 70 \\
\hline
\end{tabular}

Tabla 1: Frecuencias de la Población del estudio.

Fuente: Oficina de estadística de la UNMSM.

La selección de la muestra, como hemos señalado líneas arriba, ha sido no probabilística intencionada. Dicha representatividad se dio según criterio del investigador, es decir, fueron todos los docentes considerados como población (muestra censal).

Los datos fueron obtenidos mediante la aplicación de dos instrumentos de medición, que se pasan a detallar:

A) Para la variable gestion institucional, se elaboró un cuestionario tipo escala de Lickert.

B) Para la variable calidad del area academica universitaria, se elaboró un cuestionario tipo escala de Lickert.

\section{Validez de contenido}

La validez de contenido fue realizada por juicio de expertos, quienes verificaron si los ítems guardan relación con la teoría, la operacionalización de las variables y los objetivos planteados en la presente investigación. 


\section{Confiabilidad de instru- mentos}

El principio de confiabilidad del instrumento se establece en esta investigación mediante el coeficiente de Alfa Cronbach. Este precisa de una sola ejecución del instrumento de medición y genera cantidades que fluctúan entre cero y uno. Es ejecutado a escalas de diversos valores factibles, como consecuencia de esto se consigue utilizarlo para encontrar el nivel de confiabilidad en escalas con preguntas que tengan más de dos opciones posibles como contestaciones válidas. Su fórmula admite que encontremos el nivel de consistencia y precisión; la escala de valores que se obtiene de la confiabilidad está determinada conforme a las técnicas estadísticas.

El instrumento que mide la gestión institucional presenta un coeficiente de confiabilidad de 0.968; es decir, muy alto en su versión de 25 preguntas. Por otro lado, el instrumento que mide la calidad del área académica universitaria presenta también un alto coeficiente de confiabilidad con un alfa de Cronbach de 0.976 en su versión de 30 preguntas.

\section{Resultados}

Teniendo en cuenta que de estos dos instrumentos se obtienen resultados muy confiables, se reafirma que pueden ser aplicados a la muestra obtenida para el análisis estadístico de la presente indagación.

Respecto al diagnóstico estratégico o fase analítica del planteamiento, el $40 \%$ de los docentes menciona que es insuficiente, mientras que el $40 \%$ afirma que es regular; esto implica que para el $80 \%$ de los docentes que participaron en estas encuestas, este procedimiento no es el conveniente. Así mismo se tiene que el $12.9 \%$ manifiesta que es eficiente el nivel de desarrollo de este proceso y el $7.1 \%$ de los docentes encuestados manifiesta que es muy bueno el diagnostico estratégico.

Sobre la manera de determinación de las ideas rectoras o fase filosófica, el $35.7 \%$ de los docentes encuestados manifiestan que es inadecuado y para el $38.6 \%$ es regular, esto implica que para el $74.3 \%$ de los docentes que participaron en estas encuestas este procedimiento no es el conveniente. Así mismo se tiene que el $22.9 \%$ manifiesta que es eficiente el nivel de desarrollo de este proceso y el $2.9 \%$ de los docentes encuestados menciona que es muy bueno la manera como se define la misión, la visión, los principios y valores organizacionales.

Sobre la formulación de los objetivos estratégicos, el $35.7 \%$ de los docentes encuestados manifiesta que es inadecuado y para el $38.6 \%$ es regular. Esto implica que para el $74.3 \%$ de los docentes que participaron en estas encuestas este procedimiento no es el conveniente. Así mismo se tiene que el $20.0 \%$ manifiesta que es eficiente el nivel de desarrollo de este proceso y el $5.7 \%$ de los docentes encuestados menciona que es muy bueno la formulación de objetivos estratégicos.

Con respecto a la formulación de las estrategias y tácticas, el $34.3 \%$ de los docentes manifiesta que es inadecuado y para el $41.4 \%$ es regular, esto implica que para el $75.7 \%$ de los docentes que participaron en estas encuestas tendríamos que este pro- 
cedimiento no es el conveniente. Así mismo, el $17.1 \%$ menciona que es eficiente el nivel de desarrollo de este proceso y el $7.1 \%$ de los docentes encuestados manifiesta que es muy bueno la formulación de las estrategias y tácticas para la obtención de una buena gestión institucional.

Considerando la implantación de los planes operativos, el $40.0 \%$ de los docentes menciona que es inadecuado y para el $37.1 \%$ es regular, esto implica que para el $77.1 \%$ de los docentes que participaron en estas encuestas este procedimiento no es el conveniente. Así mismo se tiene que el $14.3 \%$ menciona que es eficiente el nivel desarrollo de este proceso y el $8.6 \%$ de los docentes encuestados afirma que es muy bueno la manera como se implanta los planes operativos organizacionales.

Sobre la gestión institucional se obtiene que para el $38.6 \%$ de los docentes es inadecuado y para el $40.0 \%$ es regular, esto implica que para el $78.6 \%$ de los docentes que participaron en estas encuestas este procedimiento no es el conveniente. Así mismo se tiene que el $17.1 \%$ menciona que es eficiente el nivel de desarrollo de este proceso y el $4.3 \%$ de los docentes encuestados afirma que es muy bueno la gestión institucional.

Respecto al desarrollo de las actividades de investigación se aprecia que para el $51.4 \%$ de los docentes estas actividades son deficientes y para el $17.1 \%$ tienen un desempeño aceptable. Esto implica que para el $68.5 \%$ de los docentes que participaron en estas encuestas este procedimiento no es el conveniente. Así mismo se tiene que el $15.7 \%$ de los docentes mencionan que es eficiente el desarrollo de estas actividades y el $15.7 \%$ afirman que es muy bueno el manejo de la línea de investigación de la universidad.

Respecto al desarrollo de las actividades lectivas se aprecia que para el $51.4 \%$ de los docentes estas actividades son inadecuadas y para el $27.1 \%$ tienen un desempeño aceptable. Esto implica que el $78.5 \%$ de los docentes que participaron en estas encuestas este procedimiento no es el conveniente. Así mismo se tiene que el $11.4 \%$ de los docentes mencionan que es eficiente el desarrollo de estas actividades y el $10.0 \%$ afirman que es muy bueno el desarrollo de las actividades lectivas en la universidad.

Sobre la responsabilidad social y extensión universitaria, el $25.7 \%$ de los docentes participantes mencionan que es inadecuado y el $35.7 \%$ dicen que es aceptable, esto implica que para el $65.4 \%$ de los docentes que participaron en estas encuestas no es conveniente la manera como se desarrolla la responsabilidad social y extensión universitaria. Así mismo se tiene que el $11.4 \%$ de los encuestados manifiestan que es eficiente el trabajo de responsabilidad social y extensión universitaria y para el $27.1 \%$ es muy bueno el trabajo desarrollado en responsabilidad social y extensión universitaria.

Respecto a la calidad del área académica universitaria, el $47.1 \%$ de los docentes participantes mencionan que es inadecuada la calidad del área académica universitaria, el $25.7 \%$ mencionan que la calidad académica es aceptable; esto implica que para el $72.8 \%$ de los docentes que participaron en estas encuestas no es significativo la calidad del área académica universitaria. Así mismo se tiene que el $18.6 \%$ afirma que es bueno el nivel y el $8.6 \%$ 
de los docentes encuestados afirma que es muy buena la calidad del área académica universitaria.

En la prueba de hipótesis general, se observa que el valor chi-cuadrado es de 51.758 puntos, mayor que 16.9289 , valor crítico de rechazo de hipótesis nula. Además, el valor de significancia es de 0.000 puntos inferior a $0.05(\mathrm{p}<0.05)$. Conforme a estos resultados obtenidos se manifiesta que hay motivos más que suficientes para rechazar la hipótesis nula y se obtendrá que es significativo el efecto del desarrollo del diagnóstico estratégico para así poder conseguir la calidad del área académica universitaria en la Escuela Académica Profesional (EAP) de Ingeniería Electrónica de la Facultad de Ingeniería electrónica y eléctrica de la UNMSM en el año 2020.

En la prueba de hipótesis especifica $\mathrm{N}^{\circ} 1$ se observa que el valor chi-cuadrado es de 50.829 puntos, mayor que 16.9289 valor crítico de rechazo de hipótesis nula. Además, el valor de significancia es de 0.000 puntos menor que $0.05(\mathrm{p}<0.05)$. Conforme a estos resultados obtenidos se manifiesta que hay motivos más que suficientes para rechazar la hipótesis nula y se obtendrá que es significativo el efecto del desarrollo del diagnóstico estratégico para así poder conseguir la calidad del área académica universitaria en la Escuela Académica Profesional (EAP) de Ingeniería Electrónica de la Facultad de Ingeniería electrónica y eléctrica de la UNMSM en el año 2020.

En la prueba de hipótesis especifica $\mathrm{N}^{\circ} 2$, se observa que el valor chi-cuadrado es de 39.317 puntos, mayor que 16.9289 valor crítico de rechazo de hipótesis nu- la, además el valor de significancia es de 0.000 puntos, menor que $0.05(\mathrm{p}<0.05)$. Conforme a estos resultados obtenidos se manifiesta que hay motivos más que suficientes para rechazar la hipótesis nula y se obtendrá que es significativo el efecto de la determinación de las ideas rectoras para así poder conseguir la calidad del área académica universitaria en la Escuela Académica Profesional (EAP) de Ingeniería Electrónica de la Facultad de Ingeniería electrónica y eléctrica de la UNMSM en el año 2020.

En la prueba de hipótesis especifica $\mathrm{N}^{\circ} 3$, se observa que el valor chi-cuadrado es de 59.759 puntos, mayor que 16.9289 valor crítico de rechazo de hipótesis nula, además el valor de significancia es de 0.000 puntos, menor que $(\mathrm{p}<0.05)$. Conforme a estos resultados obtenidos se manifiesta que hay motivos más que suficientes para rechazar la hipótesis nula y se afirma que es significativo el efecto de la formulación de los objetivos estratégicos para así poder conseguir la calidad del área académica universitaria en la Escuela Académica Profesional (EAP) de Ingeniería Electrónica de la Facultad de Ingeniería Electrónica y eléctrica de la UNMSM en el año 2020.

En la prueba de hipótesis especifica $\mathrm{N}^{\circ} 4$, se observa que el valor chi-cuadrado es de 44.093 puntos, mayor que 16.9289 valor crítico de rechazo de hipótesis nula, además el valor de significancia es de 0.000 puntos, menor que $0.05(\mathrm{p}<0.05)$. Conforme a estos resultados obtenidos se manifiesta que hay motivos más que suficientes para rechazar la hipótesis nula y se afirma que es significativo el efecto de la formulación de las estrategias y 
tácticas para así poder conseguir la calidad del área académica universitaria en la Escuela Académica Profesional (EAP) de Ingeniería Electrónica de la Facultad de Ingeniería Electrónica y eléctrica de la UNMSM en el año 2020.

En la prueba de hipótesis especifica $\mathrm{N}^{\circ} 5$, se observa que el valor chi-cuadrado es de 48.674 puntos, mayor que 16.9289 valor crítico de rechazo de hipótesis nula, además el valor de significancia es de 0.000 puntos, menor que $0.05(\mathrm{p}<0.05)$. Conforme a estos resultados obtenidos se manifiesta que hay motivos más que suficientes para rechazar la hipótesis nula y se afirma que es significativo el efecto de la implantación de los planes operativos para poder conseguir la calidad del área académica universitaria en la Escuela Académica Profesional (EAP) de Ingenieria Electrónica de la Facultad de Ingeniería Electrónica y eléctrica de la UNMSM en el año 2020.

\section{Discusión}

Dado que para el $80 \%$ de los docentes encuestados no es adecuada la atención de la etapa de diagnóstico estratégico o fase analítica del planteamiento, la Facultad debe sistematizar una mayor participación del personal docente en esta etapa del planeamiento estratégico.

Teniendo en cuenta que solo para un poco más del $25 \%$ de los docentes encuestados está entre bueno y muy bueno la determinación de las ideas rectoras o fase filosófica, la Facultad debe comprometer una mayor participación de los docentes en la determinación de la misión, la visión, los principios y valores organizacionales.

Siendo tan solo un poco más del $25 \%$ de los docentes encuestados quienes opinan que la formulación de los objetivos estratégicos o fase programática del planteamiento es bueno o muy bueno, la Facultad debe hacer más participativa la formulación de objetivos estratégicos.

Teniendo en cuenta que tan solo para cerca del $25 \%$ de los docentes encuestados, está entre bueno y muy bueno la etapa de formulación de las estrategias y tácticas o fase operativa del planteamiento, debe promover mayor participación de los docentes a fin de mejorar la gestión institucional.

Considerando que para cerca del $25 \%$ la fase de implantación de los planes operativos o fase cuantitativa del planteamiento está entre bueno y muy bueno la manera como se implanta los planes operativos organizacionales, la Facultad debe sistematizar una mejor participación de los docentes.

Teniendo en cuenta que solo un poco más del $20 \%$ de los docentes opinan que la gestión institucional está entre bueno y muy bueno el nivel desarrollo de este proceso, para mejorar la gestión institucional se deberá dar mayor importancia a este aspecto a fin de mejorar el funcionamiento de la Facultad.

Dado que tan solo para un $30 \%$ de los docentes el desarrollo de las actividades de investigación en la universidad es de nivel bueno o muy bueno, se debe incentivar un mayor compromiso a fin de que estas actividades se desarrollan de manera apropiada.

Considerando que para tan solo un $21 \%$ de los docentes encuestados, el desarrollo 
de las actividades lectivas o de docencia en la universidad son de nivel bueno o muy bueno, la Facultad debe asesorar el mejoramiento del desarrollo de estas actividades.

Siendo la opinión de tan solo un poco más del $28 \%$ de que la proyección social, está en un nivel entre bueno y muy bueno, la Facultad debe coordinar un mejoramiento de este servicio en favor de la comunidad educativa interna y externa.

Observando que tan solo un $25 \%$ de los docentes encuestados opinan que está entre bueno y muy bueno el nivel de la calidad del área académica universitaria, la Facultad debe orientar para mejorar este aspecto en favor de los estudiantes.

\section{Conclusiones}

Se ha encontrado que:

1. Es significativo el efecto de la aplicación de la gestión institucional en el logro de la calidad del área académica universitaria en la EAP de Ingeniería Electrónica de la Facultad de Ingeniería Electrónica y eléctrica de la UNMSM en el año 2020.

2. Es significativo el efecto del desarrollo del diagnóstico estratégico en el logro de la calidad del área académica universitaria en la EAP de Ingeniería Electrónica de la Facultad de Ingeniería Electrónica y eléctrica de la UNMSM en el año 2020.

3. Es significativo el efecto de la determinación de las ideas rectoras en el logro de la calidad del área académica universitaria en la EAP de Inge- niería Electrónica de la Facultad de Ingeniería Electrónica y eléctrica de la UNMSM en el año 2020.

4. Es significativo el efecto de la formulación de los objetivos estratégicos en el logro de la calidad del área académica universitaria en la EAP de Ingeniería Electrónica de la Facultad de Ingeniería Electrónica y eléctrica de la UNMSM en el año 2020.

5. Es significativo el efecto de la formulación de las estrategias y tácticas en el logro de la calidad del área académica universitaria en la EAP de Ingeniería Electrónica de la Facultad de Ingeniería Electrónica y eléctrica de la UNMSM en el año 2020.

6. Es significativo el efecto de la implantación de los planes operativos en el logro de la calidad del área académica universitaria en la EAP de Ingeniería Electrónica de la Facultad de Ingeniería Electrónica y eléctrica de la UNMSM en el año 2020.

\section{Recomendaciones}

1. La Facultad de Ingeniería Electrónica y eléctrica de la Universidad Nacional Mayor de San Marcos debe propiciar una mayor participación de los docentes para mejorar el diagnóstico estratégico o fase analítica del planteamiento, determinación de las ideas rectoras o fase filosófica, formulación de los objetivos estratégicos o fase programática del planteamiento, formulación de objetivos estratégicos, 
formulación de las estrategias y tácticas o fase operativa del planteamiento, implantación de los planes operativos o fase cuantitativa del planteamiento, a fin de mejorar la gestión institucional.

2. La Facultad de Ingeniería Electrónica y eléctrica de la Universidad Nacional Mayor de San Marcos debe promover en los docentes el mejor desarrollo de las actividades de investigación, desarrollo de las actividades lectivas o de docencia, y la proyección social, a fin de mejorar la calidad del área académica universitaria, en favor de los estudiantes.

\section{Aspectos éticos}

En el presente estudió se informó a los participantes sobre los objetivos del mismo y aceptaron participar del mismo mediante llenado de encuesta. Las encuestas fueron recopiladas de manera anónima.

\section{Agradecimientos}

Un agradecimiento a las madres y recién nacidos que participaron de este estudio e hicieron posible esta investigación y a María Lucia Bunzel por su apoyo en la recolección de los datos.

\section{Referencias}

(1) Olguín, E. y Fallas, A (2020). Percepción en la implementación de modelos de gestión educativa en instituciones de educación superior. Boletín Científico De La Escuela Superior Atotonilco De Tula, 7(13), 48-56. https://doi.org/10. 29057/esat.v7i13.5296

(2) Oseda, D. Mendivel, R. y Durán, A. (2020). Potencial de innovación y gestión institucional en la Universidad Nacional de Cañete-Perú. Sophia, colección de Filosofía de la Educación, 28(1), pp. 207-235.

(3) Márquez, M. Salazar, J. Esparza, A. y Quiñonez, F. (2019). Una mirada interna al proceso de calidad de los programas educativos en el Centro Universitario del Norte. 3C Empresa. Investigación Y Pensamiento Crítico, 88-107. Recuperado a partir de http://ojs.3ciencias.com/index. php/3c-empresa/article/view/807

(4) Zambrano, M. Ponce, E. y Santos, J. (2019). La educación de calidad en la práctica preprofesional. Opuntia Brava, 11(3), 331-341. Recuperado a partir de http://opuntiabrava.ult.edu.cu/ index.php/opuntiabrava/article/ view $/ 817$

(5) Veloz, F. Indacochea, A. y Loor, G. (2019). Estrategias de aprendizajes hacia las mejoras continuas de la calidad en la formación profesional. Opuntia Brava, 11(2), 444-457. Recuperado a partir de http://opuntiabrava.ult.edu.cu/ index.php/opuntiabrava/article/ view $/ 775$ 
(6) Leyva, L. Proenza, Y. y Mendoza, L. (2019). La calidad del aprendizaje universitario como esencia del proceso formativo que favorezca un egresado de perfil amplio. Opuntia Brava, 11(2), 228-244. Recuperado a partir de http://opuntiabrava.ult. edu.cu/index.php/opuntiabrava/ article/view/757

(7) Antón, C. (2019). La calidad educativa: unidad y diversidad. Opuntia Brava, 11(3), 342-350. Recuperado a partir de http://opuntiabrava.ult. edu.cu/index.php/opuntiabrava/ article/view/818

(8) Universidad Nacional Mayor de San Marcos (2018). Plan estratégico institucional 2019-2021. Lima: UNMSM. https://vrip.unmsm.edu.pe/ Documentos/normas/01862-18tPlan-Estrategico-Institucional.pdf

(9) Rodríguez Saif, M., \& Tardo Fernández, Y. (2018). Indicadores de evaluación del impacto de la gestión académica de posgrado para la calidad de la formación pedagógica universitaria. Opuntia Brava, 10(2), 130-143. Recuperado a partir de http: //opuntiabrava.ult.edu.cu/index. php/opuntiabrava/article/view/93

(10) Razo Abundis, L. I. Y., Iñigo Bajo, D. E. R., \& Dibut Toledo, D. C. L. S. (2017). Algunas consideraciones sobre la gestión de la calidad de la Educación Superior. Universidad Y Sociedad, 9(5), 54-62. Recupera- do a partir de https://rus.ucf.edu. $\mathrm{cu} /$ index.php/rus/article/view/711

(11) Universidad Nacional Mayor de San Marcos (2016). Plan estratégico institucional 20172019. Lima: UNMSM. https: //sistemas.unmsm.edu.pe/ocaa/ adjuntos/paginas/documentosocaa/D_PEI\%20UNMSM\%20201719.pdf

(12) Sunedu (2016) Superintendencia Nacional de Educación Superior Universitaria. Lima: https://www.gob.pe/sunedu Unesco (2015). Replantear la educación ¿Hacia un bien común mundial? https://unesdoc.unesco.org/ark: /48223/pf0000232697

(13) Ministerio de Educación (2014). Ley $\mathrm{N}^{\circ}$. 30220. Ley Universitaria. Lima: http://www.minedu. gob.pe/reforma-universitaria/pdf/ ley_universitaria.pdf

(14) León, G. (2010). Nuevos enfoques para la gestión estratégica de la innovación en las universidades. España: Universidad Politécnica de Madrid.

(15) Aristimuño, M. Guaita, W. y Rodriguez, C. (2009). Informe: "Estudio comparado de dimensiones de la evaluación institucional en instituciones de educación superior venezolanas". Venezuela: Universidad Nacional Experimental de Guayana.

(16) Ejea, G. (2008), Política Pública y Gestión Institucional en la 
educación superior: Factores organizacionales y cambio en la calidad educativa. El caso de la UAM 2001-2006.Tesis. México: Universidad Autónoma Metropolitana de México.

(17) Sovero, F. (2007). Cómo dirigir una Institución Educativa. AFA, Editores Importadores S.A. Lima.

(18) Farro, F. (2001). Planeamiento Estratégico para Instituciones Educativas de Calidad. UDEGRAF. Lima. 